
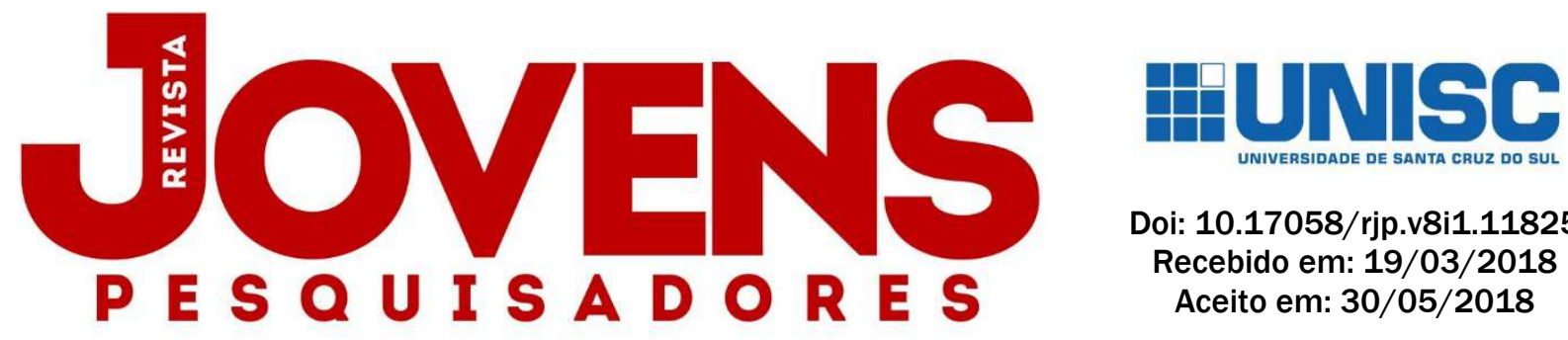

Doi: $10.17058 /$ rjp.v8i1.11825

Recebido em: 19/03/2018

Aceito em: 30/05/2018

\title{
O PERFIL SOCIOECONÔMICO E O USO DE TECNOLOGIAS DE INFORMAÇÃO E COMUNICAÇÃO (TIC) PELA AGRICULTURA FAMILIAR NO VALE DO CAÍ / RS
}

\author{
BARCELOS, L.1; RITT, D. ${ }^{2}$; DEPONTI , C. M. ${ }^{3}$; AREND, S.C. ${ }^{3}$ \\ PALAVRAS CHAVE: Agricultura. Tecnologias. Comunicação. Informação. Apropriação.
}

\section{RESUMO}

0 presente artigo refere-se ao projeto de pesquisa e de extensão denominado "O uso e a apropriação de TICs pela agricultura familiar no Vale do Caí - RS", financiado pelo edital do MCTI/CNPq. 0 objetivo deste artigo é traçar o perfil socioeconômico dos agricultores familiares do Vale Caí e verificar o uso das TIC (Tecnologias de Informação e Comunicação) pelos agricultores familiares. Foram aplicados 375 formulários de entrevistas semiestruturados com informações socioeconômicas e culturais que serviram de base para a formação do perfil dos agricultores estudados e questões relativas ao uso e à apropriação das TIC. Essas informações foram tabuladas e verificou-se que os agricultores familiares do Vale do Caí, de acordo com resultados levantados, apresentam idade média de aproximadamente 42 anos, moram com a família na propriedade rural, cultivam principalmente a silvicultura e a citricultura, pouco mais da metade tem rendimentos de até 2 salários mínimos ao mês e apresentam baixa escolaridade, basicamente o ensino fundamental incompleto. No que tange ao uso das TIC destaca-se que somente 16 propriedades não possuem bens de comunicação e de informática e em relação ao acesso à internet, constata-se que $46,1 \%$ dos entrevistados acessam diariamente a rede.

\section{THE SOCIO-ECONOMIC PROFILE AND THE USE OF INFORMATION TECHNOLOGIES AND COMMUNICATION (ICT) FOR AGRICULTURE FAMILY IN THE VALLEY OF CAÍ / RS}

KEYWORDS: Agriculture. Technologies. Communication. Information. Appropriation.

\begin{abstract}
This article refers to the research and extension project called "The use and appropriation of ICTs by family farms in the Caí Valley - RS", financed by the edict of MCTI / CNPq. The objective of this article is to outline the socioeconomic profile of the family farmers of the Caí Valley and to verify the use of ICTs (information and communication technologies) by the family farmers. A total of $\mathbf{3 7 5}$ semi-structured interview forms with socioeconomic and cultural information were used to form the profile of the farmers studied and issues related to the use and appropriation of ICT. This information was tabulated and it was verified that the family farmers of the Caí Valley, according to results obtained, present average age of approximately $\mathbf{4 2}$ years, live with the family in the rural property, cultivate mainly forestry and citriculture, just over half have incomes of up to 2 minimum wages per month and have low schooling, basically incomplete elementary education. With regard to the use of ICTs, it is noteworthy that only 16 properties do not have communication and informatics goods and in relation to access to the Internet, $46.1 \%$ of respondents access the network daily.
\end{abstract}

\footnotetext{
1 Graduada em Administração da Universidade de Santa Cruz do Sul.

2 Acadêmico do curso de Administração da Universidade de Santa Cruz do Sul.

3 Docentes do Departamento de Ciências Econômicas da Universidade de Santa Cruz do Sul.
} 


\section{INTRODUÇÃO}

O objetivo deste artigo é traçar o perfil socioeconômico e verificar o uso das TIC (Tecnologias de Informação e Comunicação) pelos agricultores familiares do Vale do Caí. Na presente pesquisa consideraram-se como TIC as tecnologias de informação e comunicação tais como a televisão, o rádio, o telefone fixo, o telefone celular, o computador de mesa, o notebook e o tablet. Consideraramse, ainda, o acesso à internet e a utilização de livros, jornais e revistas. 0 uso das TIC está relacionado com todo o processo de utilização dessas tecnologias, tais como o celular, o computador, a internet na vida cotidiana para comunicação e troca de informações.

Tal projeto conta com a parceria da EMATER/ASCAR-RS, do CETAM-EMATER, do Sindicato dos Trabalhadores Rurais do município de Montenegro-RS, das escolas rurais situadas no Vale do Caí, dos alunos dos cursos de Administração, de Ciências Contábeis, da secretaria do Campus da UNISC em Montenegro, dos alunos do Programa de Pós Graduação em Desenvolvimento Regional.

Dentre as atividades de pesquisa realizadas destacam-se:

a) reuniões com a EMATER/ASCAR-RS e Sindicato dos Trabalhadores Rurais para apresentação do projeto. Esta se caracteriza como a primeira etapa da pesquisa, ou seja, a formação e a articulação da rede de atores que participarão do Projeto. Após o primeiro contato com as organizações citadas foi calculada a amostra para seleção dos participantes do Projeto;

b) Construção do instrumento de coleta de dados com base em roteiro semiestruturado, dividido em duas partes: perfil socioeconômico e uso e apropriação das TICs;

c) realização de entrevistas através da aplicação de roteiro semiestruturado aplicado, visando à obtenção de informações socioeconômicas e culturais, além de elementos relacionados com o uso e a apropriação de TICs. 0 total de entrevistas realizado correspondeu a uma amostra de 375 agricultores familiares, caracterizado como probabilística com $95 \%$ de confiança e $5 \%$ de erro, num universo de 9.416 estabelecimentos com agricultura familiar no Vale do Caí.

d) inclusão das informações em planilha Excel para análise dos resultados;

e) inclusão das informações no SPSS;

f) análise dos dados quantitativos, através da técnica de tratamento estatístico simples (MARCONI; LAKATOS, 2006), o que possibilitou a elaboração de gráficos e de tabelas com base nas informações coletadas. A análise dos dados qualitativos ocorreu a partir da técnica de análise de conteúdo com base em Bardin (1977), constituída por três fases distintas: a organização da análise ou pré-análise, a exploração ou codificação e o tratamento dos dados e/resultados;

e) apresentação do relato da experiência em eventos científicos e publicação de artigos.

Além das atividades de pesquisa, o projeto promoveu atividades de extensão tecnológica, desenvolvidas ao longo do ano de 2017 realizadas através da criação do Núcleo de Extensão Tecnológica e Gestão Rural para a Agricultura Familiar. 0 núcleo foi criado nos municípios de 
Montenegro e de Sobradinho, atendendo agricultores familiares. 0 município de Sobradinho não faz parte do empírico do Projeto suprarreferido, mas também apresenta a agricultura familiar como fonte de dinamismo econômico. O Núcleo objetiva a recepção das demandas de agricultores familiares do Vale do Caí - RS e de Sobradinho - RS relacionadas à gestão rural.

Este artigo está dividido em duas seções, além da introdução e das considerações finais. $\mathrm{Na}$ primeira apresenta-se o perfil socioeconômico dos agricultores familiares do Vale do Caí-RS e na segunda discute-se o uso das TIC pelos agricultores familiares.

\section{PERFIL DOS AGRICULTORES FAMILIARES DO VALE DO CAÍ-RS}

Primeiramente antes de apresentar os dados dos resultados da pesquisa, trata-se dos procedimentos metodológicos adotados na coleta e na análise dos dados. 0 número de estabelecimentos de agricultores familiares na região, de acordo com Censo Agropecuário 2006, é 9.416 estabelecimentos. Foram escolhidos os 8 municípios com maior número de estabelecimentos de agricultura familiar, a saber: Montenegro, Bom Princípio, Brochier, São Sebastião do Caí, Maratá, Pareci Novo, Salvador do Sul e Feliz, totalizando uma amostra de 375 agricultores, tendo como critério para seleção a caracterização do agricultor como familiar de acordo com a Lei $n^{\circ}$ 11.326/96. Para esses agricultores foram aplicados formulários de entrevistas semiestruturados com informações socioeconômicas e culturais que serviram de base para a formação do perfil dos agricultores estudados e questões relativas ao uso e a apropriação das TIC. Essas informações foram tabuladas no Programa Statistical Package for Social Sciences (SPSS). 
Figura 1 - Municípios da região do COREDE Vale do Caí

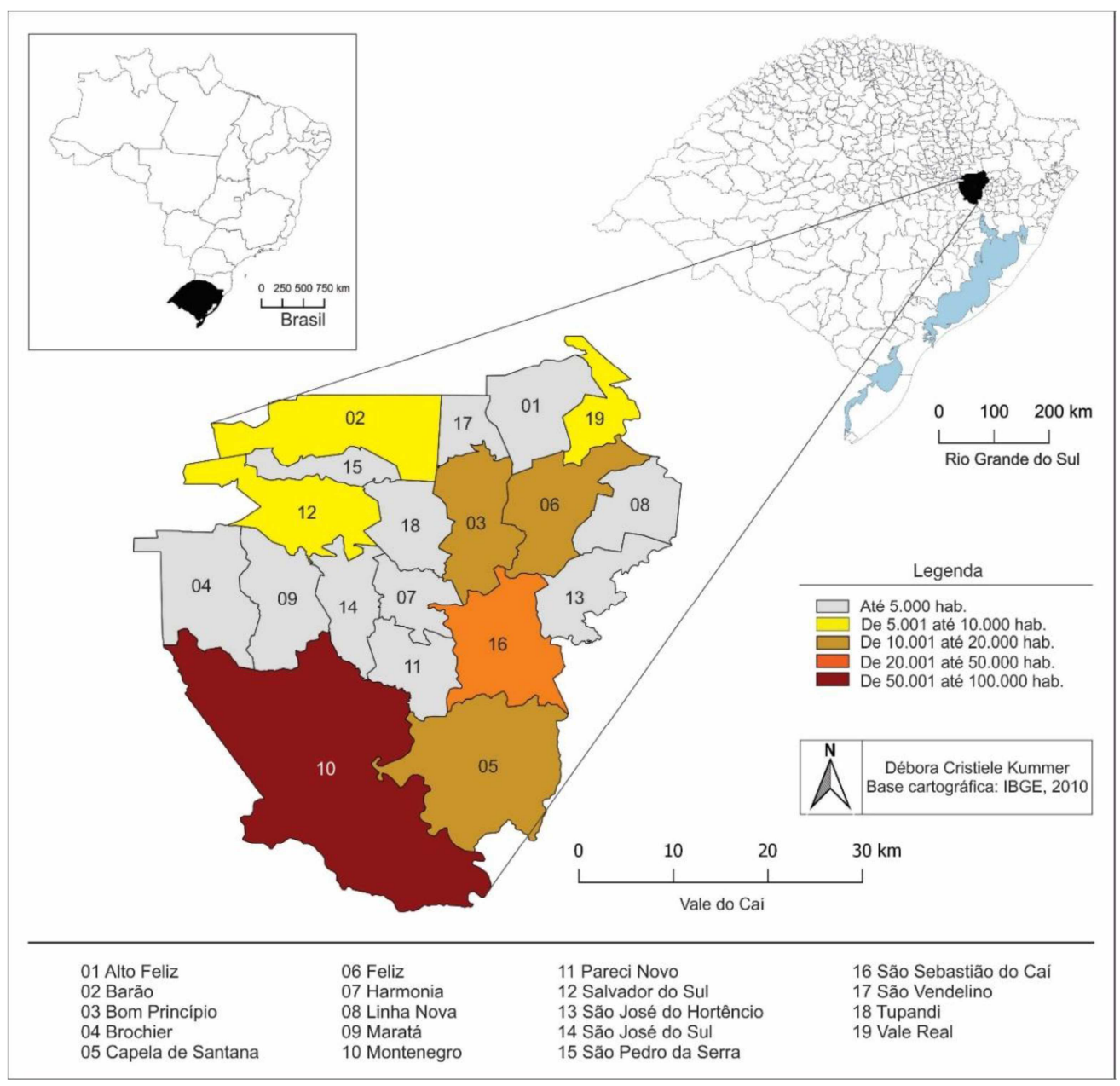

Fonte: Elaborado por Débora Cristiele Kummer (2017).

Esta seção apresenta as estatísticas descritivas da amostra de 375 propriedades rurais, sendo 211 homens e 164 mulheres entrevistadas. Com relação ao estado civil, a maior parte constitui-se de pessoas casadas (207), sendo 114 pessoas solteiras, 17 viúvos e 17 em união estável.

Tabela 01 - Distribuição das entrevistas por municípios

\begin{tabular}{c|c|c}
\hline Município & $\mathbf{N}^{\circ}$ & $\%$ \\
\hline Montenegro & 78 & 20,8 \\
\hline São Sebastião do Caí & 79 & 21,1 \\
\hline Feliz & 30 & 8,0 \\
\hline Salvador do Sul & 27 & 7,2 \\
\hline Bom Princípio & 39 & 10,4 \\
\hline Brochier & 53 & 14,1 \\
\hline Pareci Novo & 43 & 11,5 \\
\hline Maratá & 26 & 6,9 \\
\hline Total & 375 & 100
\end{tabular}

Fonte: Elaborada pelos autores a partir da pesquisa de campo (2016) 
A idade média dos entrevistados é de 41,39 anos, tendo o mais idoso 83 anos. A maior parte dos entrevistados (340) reside com familiares na propriedade; em 17 propriedades reside apenas 0 casal; 12 respondentes evidenciaram que vivem com outras pessoas que não são da família; 5 informaram que moram sozinhos e um não identificou sua condição.

Tabela 02 - Grau de escolaridade dos entrevistados

\begin{tabular}{c|c|c}
\hline Escolaridade & $\mathbf{N}^{\mathbf{0}}$ & $\%$ \\
\hline $\begin{array}{c}\text { Analfabeto ou até } 3^{\circ} \text { ano } \\
\text { fundamental }\end{array}$ & 10 & 2,7 \\
\hline Fundamental Incompleto & 184 & 49,1 \\
\hline Fundamental Completo & 61 & 16,2 \\
\hline Ensino Médio Incompleto & 25 & 6,7 \\
\hline Ensino Médio Completo & 61 & 16,2 \\
\hline Superior Incompleto & 15 & 4,0 \\
\hline Superior Completo & 13 & 3,5 \\
\hline Não informado & 6 & 1,6 \\
\hline Total & 375 & 100
\end{tabular}

Fonte: Elaborada pelos autores a partir da pesquisa de campo (2016)

A escolaridade dos produtores rurais entrevistados é baixa, pois mais da metade $(52,8 \%)$ tem até somente o Ensino Fundamental Incompleto, enquanto que apenas 15 iniciaram um curso superior e somente 13 concluíram um curso superior. Este quadro de baixa escolaridade é característica do meio rural brasileiro e a região em estudo apresenta indicadores semelhantes aos nacionais. De acordo com os dados do Censo Agropecuário 2006 aproximadamente 42\% dos estabelecimentos rurais são dirigidos por agricultores que possuem Ensino Fundamental Incompleto. Para a Região Sul estes dados tornam-se mais alarmantes, pois em torno de $78 \%$ dos estabelecimentos são dirigidos por agricultores que apresentam Ensino Fundamental Incompleto (IBGE, 2006).

A baixa escolaridade não se coloca como um empecilho nos casos em que os agricultores, embora nesta condição, não se apresentam avessos ou receosos à utilização de TIC, tanto de comunicação e de informação como de gestão. A maior dificuldade na apropriação das TIC se encontra naqueles espaços em que os agricultores revelaram diversos motivos, dentre os quais se destacam: insegurança, desconhecimento, desconfiança ou ainda o fato de não estarem dispostos a experimentar novas tecnologias e/ou processos e técnicas.

A receita das propriedades entrevistadas, em Salários Mínimos (nacional), situa-se na faixa de até três Salários Mínimos, concentrando 71,0\% das propriedades, ou seja, são propriedades com baixo ingresso mensal em termos monetários para satisfazer às necessidades, tanto das famílias, quanto de custeio e investimento na propriedade. 
Tabela 03 - Receita das propriedades

\begin{tabular}{c|c|c|c}
\hline $\mathbf{N}^{\circ}$ Salários Mínimos & $\mathbf{N}^{\circ}$ & $\%$ & \% acumulado \\
\hline$<1$ S.M. & 39 & 10,4 & 10,4 \\
\hline 1 S. M. < 2 S.M. & 130 & 34,6 & 45,0 \\
\hline 2 S. M. < 3 S.M. & 97 & 25,9 & 70,9 \\
\hline 3 S. M. < 5 S.M. & 55 & 14,7 & 85,6 \\
\hline 5 S. M. < 10 S.M. & 24 & 6,4 & 92,0 \\
\hline$>10$ S.M. & 19 & 5,0 & 97,0 \\
\hline Não informada & 11 & 3,0 & 100 \\
\hline Total & 375 & 100 & \\
\hline Fonte: elaborada pelos autores a partir da pesquisa de campo (2016)
\end{tabular}

Obs.: Salário Mínimo nacional vigente à época do levantamento: R\$ 788,00

Entre as atividades desenvolvidas nas propriedades se destaca a silvicultura, apontada por 60 entrevistados como a primeira atividade a gerar renda para a propriedade. Outras atividades de destaque são a citricultura (48 propriedades), a criação de gado de leite (23 propriedades), a olericultura (20 propriedades), a 'agricultura' (sem maiores qualificações), apontada por 29 entrevistados e a avicultura (19 propriedades). Com relação à segunda atividade principal, a maior menção foi a silvicultura, com 27 propriedades, a criação de gado de leite em 11 propriedades e a citricultura em 10 propriedades. Como terceira principal atividade o destaque é a produção de carvão, indicada por 12 entrevistados, salientando que 13 propriedades indicaram esta atividade como a principal geradora de renda em primeiro e outras oito como sendo a segunda principal atividade.

No tocante ao primeiro objetivo, verificou-se quanto:

a) ao estado civil: a maior parte constitui-se de pessoas casadas (207), sendo 114 pessoas solteiras, 17 viúvos e 17 em união estável;

b) à idade média dos entrevistados é de 41,39 anos, tendo o mais idoso 83 anos;

c) ao arranjo familiar e às condições de moradia: a maior parte dos entrevistados (340) reside com familiares na propriedade; em 17 propriedades reside apenas o casal; 12 respondentes evidenciaram que vivem com outras pessoas que não são da família; cinco informaram que moram sozinhos e um não identificou sua condição;

d) à escolaridade, em geral, os produtores rurais apresentam baixa escolaridade, sendo que mais da metade $(52,8 \%)$ possui somente o Ensino Fundamental Incompleto, enquanto que apenas 15 iniciaram e 13 concluíram um curso superior;

e) à renda, entre as famílias analisadas, constata-se que a renda se situa na faixa de até três Salários Mínimos, o que corresponde a $71 \%$ das propriedades;

f) às atividades desenvolvidas nas propriedades se destaca a silvicultura, apontada por 60 entrevistados como a primeira atividade a gerar renda para a propriedade. Outras atividades de destaque são a citricultura (48 propriedades), a criação de gado de leite (23 propriedades), a 
olericultura (20 propriedades), a 'agricultura' (sem maiores qualificações), apontada por 29 entrevistados e a avicultura (19 propriedades).

Na próxima seção analisa-se o uso das TICS pela agricultura familiar do Vale do Caí - RS.

\section{USO DE TIC PELA AGRICULTURA FAMILIAR DO VALE DO CAÍ-RS}

0 número de bens de comunicação e de informática engloba a posse de telefone celular, microcomputador de mesa (desktop), notebook e tablet. Verifica-se que somente 16 propriedades não tem nenhum destes itens, ao passo que 21 propriedades $(5,6 \%)$ possuem os quatro tipos de equipamentos.

Tabela 04 - Número de bens de comunicação e informática

\begin{tabular}{c|c|c|c}
\hline $\mathbf{N}^{\circ}$ de bens & $\mathbf{N}^{\circ}$ propriedades & $\%$ & $\%$ acumulado \\
\hline 0 & 16 & 4,3 & 4,3 \\
\hline 1 & 127 & 33,8 & 38,1 \\
\hline 2 & 154 & 41,1 & 79,2 \\
\hline 3 & 56 & 14,9 & 94,1 \\
\hline 4 & 20 & 5,3 & 99,4 \\
\hline 5 & 1 & 0,3 & 99,7 \\
\hline 6 & 1 & 0,3 & 100 \\
\hline Total & 375 & 100,0 &
\end{tabular}

Fonte: elaborada pelos autores a partir da pesquisa de campo (2016)

Desagregando estas informações por combinações possíveis de posse temos a seguinte situação: com relação à posse de celular, apenas 19 entrevistados evidenciaram não o possuir. Do total de 375 entrevistados, 129 revelaram possuir computadores, 154 possuem notebook (uma propriedade tem 3) e 50 informaram possuir tablet. Como há a possibilidade de uma propriedade ter mais de um dos equipamentos perguntados, o cruzamento de respostas apresentou 58 propriedades possuem computador e notebook, enquanto que 16 propriedades não têm nem celular, nem computador, nem notebook e tablet. A combinação mais frequente é de celular e notebook, presente em 152 propriedades (ou 40,53\% das propriedades entrevistadas).

Tabela 05 - Combinações de posse de bens de comunicação e informática

\begin{tabular}{c|c|c}
\hline Combinações & $\mathbf{N}^{\circ}$ propriedades & $\%$ \\
\hline Celular e notebook & 152 & 40,53 \\
\hline Celular e desktop & 127 & 33,87 \\
\hline Celular e tablet & 50 & 13,33 \\
\hline Notebook e desktop & 58 & 15,47 \\
\hline Notebook e tablet & 36 & 9,60 \\
\hline Desktop e tablet & 26 & 6,94
\end{tabular}

Fonte: elaborada pelos autores a partir da pesquisa de campo (2016) 
Quanto à posse de cada equipamento em particular, das 375 propriedades entrevistadas, em 356 há telefone celular (94,93\%), 154 tem notebook, 129 tem computador de mesa (desktop) e 50 possuem tablet.

A frequência diária de uso de notebook/tablet/computador é corriqueira para pouco mais da terça parte das propriedades entrevistadas (apenas $33,6 \%$ das propriedades usam diariamente), enquanto que quase a metade não usa equipamentos de informática.

Tabela 06 - Frequência diária de uso de equipamentos de informática

\begin{tabular}{c|c|c}
\hline & $\mathbf{N}^{\circ}$ propriedades & $\%$ \\
\hline Não usa & 153 & 40,8 \\
\hline Raramente & 26 & 6,93 \\
\hline Ás vezes & 70 & 18,67 \\
\hline Diariamente & 126 & 33,6 \\
\hline Total & 375 & 100 \\
\hline
\end{tabular}

Fonte: elaborada pelos autores a partir da pesquisa de campo (2016)

Com relação ao acesso à internet, 46,1\% dos entrevistados acessam diariamente a rede, enquanto que $11,7 \%$ acessam pelo menos uma vez por semana. Porém, $42,1 \%$ ou não acessam ou não costumam utilizar a Internet, quadro que reflete a pouca acessibilidade na zona rural e/ou o pouco interesse em seu uso.

Tabela 07 - Frequência de acesso à Internet

\begin{tabular}{c|c|c}
\hline Frequência & $\mathbf{N}^{\circ}$ & $\%$ \\
\hline Não acessa & 85 & 22,7 \\
\hline Diariamente & 173 & 46,1 \\
\hline Pelo menos uma vez na semana & 44 & 11,7 \\
\hline Não costumo utilizar & 54 & 14,4 \\
\hline Menos de uma vez por mês & 11 & 2,9 \\
\hline Não acessei nos últimos três meses & 6 & 1,6 \\
\hline Outra & 2 & 0,5 \\
\hline Total & 375 & 100,0
\end{tabular}

Fonte: elaborada pelos autores a partir da pesquisa de campo (2016)

0 acesso diário à internet mais frequente é de até uma hora diária $(29,6 \%)$, mas considerando que $37,9 \%$ não acessam a internet (142 propriedades) e que 150 propriedades não têm nem computador de mesa nem notebook, o uso e disseminação de conhecimentos e tecnologias pela rede mundial de acesso à informação representa um obstáculo a ser vencido na região. 
Tabela 08 - Horas diárias de acesso à Internet

\begin{tabular}{c|c|c}
\hline Frequência & $\mathbf{N}^{\mathbf{0}}$ & $\%$ \\
\hline Não acessam & 142 & 37,9 \\
\hline Até 1h & 111 & 29,6 \\
\hline Entre 1h e 2h & 48 & 12,8 \\
\hline Entre 2h e 3h & 37 & 9,9 \\
\hline Entre 3h e 4h & 10 & 2,7 \\
\hline Mais de 4h & 27 & 7,2 \\
\hline Total & 375 & 100
\end{tabular}

Fonte: elaborada pelos autores a partir da pesquisa de campo (2016)

As atividades preferidas quando do acesso à Internet foram identificadas genericamente como "pesquisas" (126 citações), seguidas pela busca por "notícias" (100 citações), "músicas e filmes" (78 citações), "jogos" (59 citações), "comunicações instantâneas" (58 citações) e "e-mail", com 55 citações. Como era solicitada a indicação de três atividades, a soma de respostas excede a 375 (total de propriedades entrevistadas).

Tabela 09 - Funcionalidades mais utilizadas na Internet

\begin{tabular}{c|c}
\hline Funcionalidade & $\mathbf{N}^{\mathbf{0}}$ \\
\hline Sites de relacionamento & 36 \\
\hline Comunicações instantâneas & 58 \\
\hline Músicas e filmes & 78 \\
\hline Jogos & 59 \\
\hline Pesquisas & 126 \\
\hline E-mail & 55 \\
\hline Notícias & 100 \\
\hline Salas de bate papo & 16 \\
\hline Outro & 18
\end{tabular}

Fonte: elaborada pelos autores a partir da pesquisa de campo (2016)

Quanto ao segundo objetivo verificou-se que somente 16 propriedades não possuem bens de comunicação e de informática (telefone celular, microcomputador de mesa/desktop, notebook e tablet), ao passo que 20 propriedades (5,3\%) possuem os quatro tipos de equipamentos.

No que se refere especificamente à posse de celular, apenas 19 entrevistados evidenciaram não o possuir. Do total de 375 entrevistados, 129 revelaram possuir computadores, 154 possuem notebook e 50 informaram possuir tablet. Como há a possibilidade de uma propriedade ter mais de um dos equipamentos perguntados, o cruzamento de respostas apresentou 58 propriedades possuem computador e notebook, enquanto que 16 propriedades não têm nem celular, nem computador, nem notebook e tablet. A combinação mais frequente é de celular e notebook, presente em 152 propriedades (ou 40,53\% das propriedades entrevistadas).

Quanto à posse de cada equipamento em particular, das 375 propriedades entrevistadas: 
a) em 356 há telefone celular (94,93\%), 154 tem notebook, 129 tem computador de mesa (desktop) e 50 possuem tablet;

b) a frequência diária de uso de notebook/tablet/computador é corriqueira para pouco menos da terça parte das propriedades entrevistadas (apenas 33,6\% das propriedades usam diariamente), enquanto que quase a metade não usa equipamentos de informática;

c) ao acesso à internet, constata-se que $46,1 \%$ dos entrevistados acessam diariamente a rede, enquanto que $11,7 \%$ acessam pelo menos uma vez por semana. Porém, 42,1\% ou não acessam ou não costumam utilizar a Internet, quadro que reflete a pouca acessibilidade na zona rural e/ou o pouco interesse em seu uso.

0 acesso diário à internet mais frequente é de até uma hora diária (29,6\%), mas considerando que 37,9\% não acessam a internet (142 propriedades) e que 150 propriedades não têm nem computador de mesa nem notebook; d) as atividades preferidas quando do acesso à Internet foram identificadas genericamente como "pesquisas" (126 citações), seguidas pela busca por "notícias" (100 citações), "músicas e filmes" (78 citações), "jogos" (59 citações), "comunicações instantâneas" (58 citações) e "e-mail", com 55 citações. Como era solicitada a indicação de três atividades, a soma de respostas excede a 375 (total de propriedades entrevistadas).

\section{CONSIDERAÇÕES FINAIS}

A experiência resultante da pesquisa realizada com agricultores familiares através do Projeto "O uso e a apropriação de TICs pela agricultura familiar no Vale do Caí - RS" evidencia elementos importantes, tais como:

A heterogeneidade da agricultura familiar no que se refere ao processo de produção (acesso aos mercados, capacidade de geração de renda e de acumulação) e às características dos agricultores (grau de escolaridade, composição familiar, diversidade produtiva das propriedades no tocante ao tamanho, aos tipos de cultivo e de criações disponibilidade de recursos), elementos que dificultam uma generalização quanto ao modelo de gerenciamento compatível com as distintas características.

O nível educacional e a idade avançada dos agricultores aliada à cultura de não realizar registros escritos dificulta a implantação de práticas de gerenciamento; necessidade de maior valorização dos agricultores com relação às atividades de campo em detrimento das atividades de gestão.

A dificuldade dos agricultores em acompanhar e considerar a evolução do mercado e as alterações nos hábitos de consumo, ultrapassando a consideração de suas atividades de forma desvinculada dos demais segmentos da cadeia produtiva ou dos próprios hábitos dos consumidores. 
Assim, concluiu-se que os agricultores familiares do Vale do Caí apresentam acesso às TICs, embora o uso e a disseminação de conhecimentos e de tecnologias pela rede mundial de acesso à informação representa obstáculo a ser vencido na região.

\section{REFERÊNCIAS}

AREND, S. C.; DEPONTI, C. M.; KIST, R. B. B. 0 uso de TIC pela agricultura familiar no território do Citros: Vale do Caí-RS. Informe Gepec (Impresso), v. 20, p. 71-84, 2016.

CENTRO DE ESTUDOS SOBRE AS TECNOLOGIAS DA INFORMAÇÃO E DA COMUNICAÇÃO - CETIC.BR. Pesquisas e Indicadores sobre TIC a domicílios, 2016. Disponível em: <http://data.cetic.br/cetic/>. Acesso em: 12 de outubro de 2017.

CETIC. Pesquisa sobre o uso das tecnologias de informação e comunicação nos domicílios brasileiros [livro eletrônico]: TIC domicílios 2015. São Paulo: Comitê Gestor da Internet no Brasil, 2016.

DEPONTI, C. M. As "agruras" da gestão da propriedade rural pela agricultura familiar. REDES, Revista do Desenvolvimento Regional, Santa Cruz do Sul, v. 19, ed. especial, 2014, p. 9-24.

DEPONTI, C. M. Intervenção para o desenvolvimento rural: o caso da extensão rural pública do Rio Grande do Sul. Porto Alegre, 2010. 276 f. Doutorado (Desenvolvimento Rural) - Universidade Federal do Rio Grande do Sul, Porto Alegre, 2010.

DEPONTI, C. M.; KIST, R.B.B.; AREND, S. Desenvolvimento regional e agricultura familiar: o uso e a apropriação das Tics no Vale do Caí - RS, Desenvolvimento Regional em debate, v. 5, n. 2, p. 170187, jul./dez. 2015.

DEPONTI, C. M.; KIST, R. B. B.; AREND, S. C.; TIC e Agricultura Familiar: uma experiência de pesquisa e de extensão tecnológica no território do Citros-RS. In: Anais Seminário Internacional de Desenvolvimento Regional, p., 2017.

DEPONTI, C.M., AREND, S. C., KIST, R. B.B. Relatório de Pesquisa. 0 uso e a apropriação de Tecnologias de Informação e de Comunicação (TICs) pela Agricultura Familiar no Vale do Caí - RS. Santa Cruz do Sul. 2017.

DEPONTI, C. M., KIST, R. B. B., MACHADO, A. As Inter-relações entre as Tic e a Agricultura Familiar. Revista Eletrônica Competências Digitais para a Agricultura Familiar. RECODAF. V.03, n. 01. 2017. P. 4-23.

DEPONTI, C. M., FELIPPI, A. C. T., DORNELLES, M. Os usos e as apropriações das Tics na agricultura familiar em regiões do sul do Brasil. Anais do VII Seminário Internacional sobre Desenvolvimento Regional. Globalização em Tempos de Regionalização - Repercussões no Território, Santa Cruz do Sul, RS, Brasil, 2015.

DHM por município. Disponível em: <http://www.atlasbrasil.org.br/2013/pt/ranking>. Acessado em 09 de Abril de 2016.

FREIRE, M. Comunicação de Informações tecnológicas para o meio rural. Ci Inf. 13 (1): jan-jun, 1984, p. 67-71.

FUNDAÇÃO DE ECONOMIA E ESTATÍSTICA - FEE Porto Alegre. Disponível em: <http://www.fee.rs.gov.br/perfil-socioeconomico/coredes/> Acesso em: 11 mar. 2017

GOVERNO DO ESTADO DO RIO GRANDE DO SUL. Lei ${ }^{\circ} 10.283$, de 17 de outubro de 1994. Dispõe sobre a criação, estruturação e funcionamento dos Conselhos Regionais de Desenvolvimento e dá outras providências. Porto Alegre, Palácio Piratini, 1994.

GOVERNO DO ESTADO DO RIO GRANDE DO SUL. Decreto $n^{\circ} 35.764$, de 28 de dezembro de 1994. Regulamenta a Lei $n^{\circ} 10.283$, de 17 de outubro de 1994, que criou os Conselhos Regionais de Desenvolvimento. Porto Alegre, Palácio Piratini, 1994a.

GOVERNO DO ESTADO DO RIO GRANDE DO SUL. Secretaria do Planejamento, Mobilidade e Desenvolvimento Regional. Perfil Socioeconômico Corede Vale do Caí. Porto Alegre: Departamento de Planejamento Governamental, 2015.

INSTITUTO BRASILEIRO DE GEOGRAFIA E ESTATISTICA (IBGE). Censo Demográfico. Brasília: Gov. $\begin{array}{lllll}\text { Federal, } 2010 . & \text { Disponivel } & \text { Censo } & 2010 .\end{array}$ 
<http://www.censo2010.ibge.gov.br/sinopse/index.php?dados=29\&uf=43>. Acessado em: 09 de Abril de 2016.

INSTITUTO BRASILEIRO DE GEOGRAFIA E ESTATISTICA (IBGE). Cidades. Disponível em: <http://www.cidades.ibge.gov.br>. Acessado em: 09 de Abril de 2016. Acessado em 08 de Abril de 2016.

INSTITUTO BRASILEIRO DE GEOGRAFIA E ESTATÍSTICAS. IBGE. Sinopse do Censo Demográfico 2010. Rio de Janeiro, IBGE, 2011.

MAIA, Roousiley C. M. Redes Cívicas e Internet: do ambiente informativo denso às condições da deliberação pública. In: EISENBERG, José; CEPIK, Marco (orgs.). Internet e Política: teoria e prática da demcoracia eletrônica. Belo Horizonte: Editora UFMG, 2002, p. 46-72.

ORGANIZAÇÃO DAS NAÇÕES UNIDAS - ONU. Information Economy Report: digitalization, trade and development. Switzerland: United Nations Conference on Trade and

Development - UNCTAD; 2017. Sales No. E.17.II.D.8. Disponível em: <http://unctad.org/en/PublicationsLibrary/ier2017_en.pdf>. Acesso em: 12 de outubro de 2017.

PERFIL SOCIOECONÔMICO COREDE VALE DO CAÍ. Porto Alegre, novembro de 2015. Disponível em: <http://planejamento.rs.gov.br/upload/arquivos/201512/15134136-20151117104014perfisregionais-2015-vale-do-cai.pdf> Acesso em: 11 mar. 2017

PESQUISA NACIONAL POR AMOSTRA DE DOMICÍLIOS (PNAD). Acesso à Internet e Posse de Telefone Móvel Celular para Uso Pessoal $2015 . \quad$ Disponível em: <https://biblioteca.ibge.gov.br/visualizacao/livros/liv98887.pdf>. Acesso em: 12 de outubro de 2017.

PREFEITURA MUNICIPAL DE MONTENEGRO. Disponível em: <http://www.montenegro.rs.gov.br/> Acesso em: 26 abr. 2017

REDIN, E.; SILVEIRA, P. R. C., GUIMARÃES, G. M., SANTOS, V. F. Juventude rural e novas formas de sociabilidade mediadas pelas TICs. Signos do consumo. São Paulo , v.5, n.2, 2013. p. 225-244, dez. 2013.

<http://www.revistas.usp.br/signosdoconsumo/article/viewFile/76390/80099>. Acesso em: 24 nov.2016.

SCHWARTZ, C. A recepção das Tecnologias de Informação e Comunicação entre os agricultores familiares de Santa Maria, Rio Grande do Sul. Dissertação de Mestrado. Santa Maria: UFSM. (2007). 